Published in final edited form as:

J Cancer Surviv. 2016 February ; 10(1): 103-112. doi:10.1007/s11764-015-0456-9.

\title{
Physical inactivity and risk of poor quality of life among elderly cancer survivors compared to women without cancer: The lowa Women's Health Study
}

\author{
Cindy K. Blair, PhD ${ }^{1}$, Kim Robien, PhD, RD², Maki Inoue-Choi, PhD, RD ${ }^{3}$, Wendy Rahn, \\ $\mathrm{PhD}^{4}$, and DeAnn Lazovich, PhD $^{1,5}$ \\ ${ }^{1}$ Division of Epidemiology \& Community Health, University of Minnesota, Minneapolis, MN \\ ${ }^{2}$ Department of Exercise and Nutrition Sciences, Milken Institute School of Public Health, George \\ Washington University, Washington, DC \\ ${ }^{3}$ Division of Cancer Epidemiology and Genetics, National Cancer Institute/National Institute on \\ Minority Health and Health Disparities, Washington, DC \\ ${ }^{4}$ Department of Political Science, University of Minnesota, Minneapolis, MN \\ ${ }^{5}$ Masonic Cancer Center, University of Minnesota, Minneapolis, MN
}

\section{Abstract}

\begin{abstract}
Purpose-Few studies have examined lifestyle factors and quality of life (QOL) in cancer survivors compared to a cancer-free group. Compared to active cancer-free women, we examined the association between physical inactivity and QOL in elderly cancer survivors and similar-aged women without cancer.
\end{abstract}

\begin{abstract}
Methods-Participants included 1,776 cancer survivors and 12,599 cancer-free women enrolled in the Iowa Women's Health Study in 1986 who completed the SF36 QOL assessment in 2004 (ages 73-88 years). The odds of poor QOL were computed for each SF36 subscale ( $>0.5 \mathrm{SD}$ below mean score of cancer-free women) associated with physical inactivity (moderate-vigorous activity <once/week) between 4 groups based on the cross-classification of cancer history (no/yes) and physical inactivity (no/yes)(referent group=active cancer-free women).
\end{abstract}

Results-Compared with the referent group, inactive cancer survivors were significantly more likely to report poor QOL for each SF36 subscale (Odds Ratios:1.8 to 4.7), independent of age, comorbidity, BMI, and diet quality. The greatest odds for poor QOL occurred for general health, vitality, and physical function. These increased odds occurred regardless of whether survivors were inactive at both baseline (1986) and follow-up or became inactive sometime after baseline. Among physically active women, cancer survivors had similar QOL as cancer-free women.

Correspondence: Cindy Blair, PhD, 1300 South Second Street, Suite 300, Minneapolis, Minnesota, 55454, Telephone: 612-625-4542/ Fax: 612-624-0315, ; Email: blair016@umn.edu.

CONFLICT OF INTEREST

The authors declare that they have no conflicts of interest or financial disclosures. 
Conclusion-These findings provide evidence on the importance of leisure-time physical activity in older women and support the need for interventions to help older women maintain or regain a physically active lifestyle.

Implications for Cancer Survivors-Survivors who remain or become physically active as they age report better mental and physical QOL.

\section{Keywords}

quality of life; physical activity; survivorship; cohort; cancer survivors

\section{INTRODUCTION}

Currently, there are over 13.7 million cancer survivors in the United States, with $60 \%$ over the age of 65 years [1]. Almost two-thirds of cancer survivors have survived five years or more since diagnosis; forty percent have survived ten years or more [1]. With the increase in survival time, the quality of years lived after a cancer diagnosis has increased in importance. Quality of life (QOL) is an important endpoint beyond cancer recurrence and survival, which shifts the focus from how long a cancer patient survives to how well a patient survives. Data from national cross-sectional surveys indicate that cancer survivors have worse QOL compared with non-cancer controls [2-4]. Cancer survivors generally report worse physical QOL within one to two years of diagnosis when they are likely undergoing treatment $[2,3]$. Survivors who are obese, physically inactive, eat an unhealthy diet or who have greater comorbidity are more likely to report worse QOL compared to survivors with healthy behaviors and few comorbidities [5-12]. Studies that have examined the effects of multiple health behaviors on QOL have concluded that physical activity had the strongest association [9, 13].

Despite the numerous benefits of physical activity on health, including lower rates of allcause mortality, coronary heart disease, and type 2 diabetes [14], only one-quarter to onethird of adults aged 40 years and older meet the recommended guidelines of 150 minutes of moderate to vigorous physical activity per week [15-17]. Despite the teachable moment of a cancer diagnosis and the reported interest in improving health behaviors [18], cancer survivors are no more likely to meet physical activity recommendations, and in some cases are less likely, compared to the general population [15-17, 19]. Among cancer survivors, studies have described a change in physical activity across the cancer trajectory, with levels decreasing after diagnosis, increasing after treatment completion, but not always returning to pre-diagnosis levels [20, 21]. Evidence from observational studies suggests that cancer survivors who engage in higher levels of physical activity report better QOL for many domains $[6,8,22]$, especially physical domains such as physical functioning [5, 7, 8, 10, 23], compared with inactive survivors. Evidence from randomized clinical trials suggests that exercise after a cancer diagnosis, even among older sedentary survivors, can improve QOL, especially physical QOL [24, 25].

While many studies have examined physical activity and QOL in cancer survivors, many research gaps remain. The majority of studies have focused on middle-aged and recently diagnosed ( $<5$ years since diagnosis) cancer survivors. Individuals currently undergoing or 
recently completing therapy and individuals diagnosed with late-stage disease, and thus worse prognosis, are likely to be less physically active and report worse QOL compared to long-term survivors. Most studies have focused on quantifying and comparing the average QOL scores, which has provided meaningful and important information; however, by identifying individuals with the worst QOL, interventions can be targeted to this subgroup. Few studies of physical activity and QOL have prospectively ascertained physical activity prior to the cancer diagnosis. Furthermore, no studies of physical activity and QOL have included a similar-aged, non-cancer comparison group, and thus it is difficult to separate the effects of cancer, aging, and comorbidity. We addressed these gaps by examining the degree to which physical inactivity is associated with poor QOL among older, long-term female cancer survivors compared to similar-aged women without cancer.

\section{MATERIALS AND METHODS}

\section{Study Design and Participants}

The Iowa Women's Health Study (IWHS) is a prospective cohort that started in 1986 to examine risk factors for cancer incidence and mortality in post-menopausal women [26]. A total of 41,836 women, aged 55 to 69 years, were enrolled. Exposure data were updated via follow-up surveys in 1987, 1989, 1992, 1997, and 2004. The 2004 questionnaire, which included a QOL assessment, was completed by 20,844 women among the 30,232 women who were alive at that time (69\% response rate). Among the 20,844 women who returned the 2004 questionnaire, women with prevalent cancer at baseline $(\mathrm{N}=1,511)$, who did not complete the QOL assessment $(\mathrm{N}=1,863)$, or whose questionnaire was completed by proxy $(\mathrm{N}=328)$ were excluded from analyses. To reduce the effect of cancer treatment on QOL and health behaviors, women diagnosed within two years of the QOL assessment $(\mathrm{N}=587)$ or who reported currently receiving cancer treatment $(\mathrm{N}=281)$ were excluded. To minimize the possibility for poor QOL due to impending death, women who died within one year of completing the 2004 questionnaire $(\mathrm{N}=411 ; 96.4 \%$ due to a health related issue) were excluded. Further exclusions were made for women who did not provide answers to questions on physical activity ( $\mathrm{N}=163)$ and current weight $(\mathrm{N}=280)$, or with incomplete or implausible data on diet ( $>30$ blank food items or dietary intake $<600$ or $>5,000 \mathrm{kcal} /$ day; $\mathrm{N}=1,045$ ), resulting in 14,375 women in the analytic cohort.

Incident cancer diagnoses between 1986 and 2002 were ascertained through linkage to the State Health Registry of Iowa, which participates in the Surveillance, Epidemiology, and End Results (SEER) program. Age and date of cancer diagnosis, cancer site, and stage of disease were obtained from the registry. Deaths were ascertained through linkage to the Iowa health registry and the National Death Index.

\section{Outcomes and Measures}

QOL-The Medical Health Outcomes Study Short Form-36 item survey (SF-36, version 2) was used to assess health-related QOL in 2004 (the only year that QOL was assessed). The SF-36 is comprised of 8 scales, which measure the following constructs: physical functioning, role physical, bodily pain, general health, mental health, role emotional, social functioning and vitality. The SF-36 instrument is valid and reliable for use in healthy and 
chronically ill adults [4, 27]. Higher scores indicate better functioning and well-being. For each of the 8 scales, poor QOL was defined as $>0.5$ standard deviations below the mean score of women without cancer. A one-half standard deviation was chosen since it has been reported to represent the minimally clinically important difference for this QOL instrument [28].

Physical Inactivity-Leisure-time physical activity was assessed at baseline and reassessed in 2004 with two questions regarding the frequency (ranging from rarely/never to more than four times per week) of moderate activities (e.g., bowling, golf, light sports/ physical exercise, gardening, taking long walks) and vigorous activities (e.g., jogging, racket sports, swimming, aerobics). Duration of activity was not assessed. Women who reported participating in moderate or vigorous physical activity less than once per week were categorized as physically inactive. Change in physical activity status was based on the crossclassification of activity status at baseline (1986; inactive vs. active) and activity status at the 2004 follow-up (inactive vs. active): stayed active (referent), became active, became inactive, and stayed inactive.

Health, demographic, and lifestyle factors-Age and education were obtained from the baseline questionnaire. Medical conditions were assessed at baseline and at each followup. The total number of comorbidities is the sum of the self-reported conditions (heart attack, heart disease, diabetes, stroke, hypertension, rheumatoid arthritis, hip fracture, and Parkinson disease). Lifestyle factors were reassessed in 2004 and used in this analysis, including body mass index (BMI; $\mathrm{kg} / \mathrm{m}^{2}$ ), smoking status, and diet via a 127 -item Harvard food frequency questionnaire [29]. A diet quality score was computed as the sum of adherence ( $0=$ non-adherent, $0.5=$ partially adherent, $1=$ =adherent) to the World Cancer Research Fund/American Institute for Cancer Research dietary recommendations for cancer prevention [30], which included six indicators: avoid high-sugar beverages; high fruit and vegetable intake, and dietary fiber intake; and limited consumption of red and processed meat, alcohol, and sodium [31].

\section{Statistical Analyses}

For the cross-sectional analysis (2004 data), logistic regression was used to compute the odds of poor QOL for each of the eight SF-36 scales associated with physical inactivity between four groups, based on the cross-classification of cancer history (yes/no) and physical inactivity (yes/no). Active women without cancer served as the referent group. In an exploratory analysis, we also compared the odds of poor QOL by change in physical activity status (from 1986 to 2004) among cancer-free women and cancer survivors, stratified on median time between diagnosis and 2004 follow-up (9.4 years). Cancer-free women who were active at both time points (1986 and 2004) served as the referent group. All models were adjusted for age, BMI, diet quality score, and number of comorbidities. Further adjustment for education, smoking status, or alcohol intake had negligible influence on the estimates, and thus these variables were not included in the final model. Interaction between cancer history and physical inactivity was assessed by including a cross-product term in the model and evaluated using the Wald test. Statistical significance for all analyses was defined as $p<0.05$. 


\section{RESULTS}

The analytic cohort consisted of 1,776 women diagnosed with incident cancer during 19862002 and 12,599 women who remained cancer free from 1986-2004, who completed the QOL assessment in 2004, and were still alive one year later. Compared with the cohort at baseline or with non-respondents to the 2004 questionnaire, women who were included in this analysis were significantly younger, more educated, more likely to report excellent health status and fewer co-morbidities at baseline, and less likely to consume alcohol (Supplemental table, online only; similar data previously described [32]). Among those who completed the 2004 questionnaire, including the QOL assessment, women who were included in the analyses were significantly younger ( $47 \%$ vs. $35 \%$ ), more educated (some college; $34 \%$ vs. $27 \%$ ), and more likely to report better QOL (all 8 scales) compared to women who were excluded (data not shown).

In our analytic cohort, the mean age was $78.6 \pm 3.9$ years, $58 \%$ were overweight or obese, and $38 \%$ had two or more non-cancer comorbidities. The most common comorbidities were hypertension (62\%), heart disease (25\%), and rheumatoid arthritis (17\%). Women who reported being physically inactive in 2004 were older and had less education, lower diet quality scores, higher BMI, and more comorbidities compared with women who were active (Table 1). Among physically inactive women, cancer survivors and cancer-free women were similar with respect to demographic and lifestyle factors. Similarly, physically active women had similar characteristics, regardless of cancer history.

Among cancer survivors, the average age at diagnosis was 69 years. Almost one-half were breast cancer survivors and one-third were colorectal or gynecologic cancer survivors (Table 2). Eighty percent were long-term survivors ( 25 years since diagnosis) and $77 \%$ were diagnosed with early stage disease.

Compared with physically active cancer-free women (referent group), active cancer survivors were not at increased risk of poor QOL for any of the eight SF-36 scales after adjusting for age, BMI, diet quality, and number of comorbidities (Table 3). In contrast, the two physically inactive groups had a statistically significant 1.8 to 4.7 fold higher odds of poor QOL, with similar risk between inactive cancer-free women and inactive cancer survivors compared with the referent group. The greatest odds of poor QOL occurred for general health (OR [95\% CI]: cancer-free $=3.0$ [2.7-3.2], cancer-survivors=3.4 [2.9-4.0]), vitality (cancer-free $=3.1$ [2.8-3.4], cancer-survivors $=3.3$ [2.9-3.9]), and physical function (cancer-free $=4.0$ [3.7-4.4], cancer-survivors $=4.7$ [4.0-5.5]). There was no evidence of interaction between cancer history and physical inactivity status on the odds of poor QOL for any of the scales (all p-values >0.17).

The association between poor QOL and change in physical activity status (between 1986 and 2004), stratified on time between diagnosis and QOL assessment in 2004 (median cutpoint of 9.4 years), are presented in Table 4. Between 1986 and 2004, the percentage of cancer-free women who stayed active, became active, became inactive, and stayed inactive was $38 \%, 19 \%, 18 \%$, and $24 \%$, respectively. The corresponding percentage of cancer survivors was $34 \%, 17 \%, 22 \%$, and $28 \%$. Compared with cancer-free women who were 
active at both time points (referent group), women whose status changed from active to inactive had a statistically significant 1.6 to 5.4-fold higher odds of poor QOL for all domains among both cancer survivors and cancer-free women. Among women who became inactive, survivors more proximal to diagnosis ( $\$ .4$ years) had a greater odds of poor QOL compared with survivors more distal from diagnosis (>9.4 years; all QOL domains) and cancer-free women (most QOL domains). Among women who were inactive at both time points, survivors more distal from diagnosis generally had the highest odds of poor QOL (ORs ranging from 2.1 to 6.0). In contrast, compared with the referent group, cancer survivors who stayed active were not at increased risk of poor QOL, regardless of time since diagnosis.

\section{DISCUSSION}

Compared with physically active cancer-free women, active cancer survivors were not at risk for poor mental and physical QOL. In contrast, physically inactive women reported significantly worse QOL, regardless of cancer history. Inactive cancer survivors had the highest odds of poor general health, vitality, and physical function. Among survivors more proximal to diagnosis ( $\$ 9.4$ years), change in status from inactive to active during the follow-up period conferred the highest odds of poor QOL. Among survivors more distal from diagnosis ( $>9.4$ years), being physically inactive at both time points conferred the highest odds of poor QOL.

Prior studies of physical activity and QOL have lacked a non-cancer comparison group; however, there is general consensus that long-term cancer survivors who are more physically active after diagnosis report better QOL, especially physical QOL, compared to less active survivors [5, 6, 8-10]. An important and novel finding in the current study is the similar odds of reporting poor mental and physical QOL between inactive cancer survivors and inactive cancer-free women for most of the QOL scales after accounting for age, BMI, diet, and number of comorbidities. Additionally, active cancer survivors had similar low odds for poor QOL as active cancer-free women.

The strong association between physical inactivity and poor physical function is of particular importance, since cancer survivors are at increased risk of physical functional impairment due to age- and treatment-related morbidity $[4,33]$. In this study, inactive cancer survivors had nearly 5 -fold higher odds of poor physical function compared with women who maintained a physically active lifestyle, regardless of cancer history. Cancer survivors who were inactive at baseline, but became active afterwards had one-third the odds of poor QOL as survivors who became or stayed inactive. These results provide further evidence that becoming active later in life can alter the trajectory of physical functional decline, as demonstrated in clinical trials [24].

A limitation of our study is self-reported physical activity, which only included frequency of moderate-vigorous recreational activity. Duration of activity was not assessed, which could have led to misclassification of physically inactive status if women reporting infrequent activity ( $<1$ day per week) were active for longer duration than women reporting more frequent activity but for shorter duration. At the time of follow-up, the women were 73 to 88 
years of age, and thus may have been participating in light-intensity activities (e.g., light gardening, leisurely walking), which were not captured by the questionnaire, or reporting moderate activity which in reality may have been performed at a lower intensity level (e.g., brisk vs. leisurely walking). The majority of prior studies that examined the association between physical activity and QOL have defined being physically active based on the frequency and duration of moderate-vigorous intensity activities $[5,7,10]$, typically based on meeting the public health activity guidelines ( $>30$ minutes/day for $\geq 5$ days/week) $[7,10]$. However, emerging evidence suggests that light-intensity activities, including non-exercise activities, or being insufficiently active (some physical activity, but less than the guidelines) also confers beneficial effects on mental and physical QOL in older survivors [7, 8, 34]. This is consistent with the Physical Activity Guidelines Advisory Committee Report that concluded that "some physical activity is better than none" [14]. Since walking and nonexercise activities performed at a light- to moderate-intensity level may be more common in the elderly survivorship population or those with moderate- to severe comorbidities, more research is needed to inform the effects of these types of activities on QOL.

The timing of the physical activity assessment in our cohort (1986 and 2004) did not allow us to precisely examine pre- vs. post-diagnosis activity level or reasons for change in activity. Nevertheless, our analysis of change in activity status over 18 years of follow-up and QOL sheds some light on the results of our cross-sectional analysis as to whether physical inactivity influenced QOL or whether poor QOL affected physical activity level. Even though there was a significantly increased odds of poor QOL reported by women who became active at some point during follow-up, these estimates were less than the estimates for women who became inactive or stayed inactive during this time frame. This suggests that adopting a more active lifestyle later in life may be beneficial for QOL. Unfortunately, few studies have evaluated the effect of physical activity level prior to diagnosis on postdiagnosis QOL, and the results are inconsistent [7, 23, 35]. The inconsistency is likely due to differences in study design including the retrospective ascertainment of pre-diagnosis physical activity, focus on recently diagnosed cancer survivors ( $<5$ years since diagnosis), and differences in cancer type, QOL instrument, and categorization of physical activity. Evidence is more consistent among studies examining change in activity level since diagnosis on subsequent QOL. Cancer survivors who increased their activity level after diagnosis reported better QOL and those with decreased activity levels reported worse QOL $[6,22,23,36]$.

Additional limitations to our study include survival bias, as our focus was on older long-term cancer survivors, an understudied group. Inclusion in this analysis was reliant on being mentally, cognitively, and physically healthy enough to complete a lengthy follow-up survey, including a full food frequency questionnaire, and thus may not be representative of the general geriatric population. Additionally, our questionnaire did not allow us to fully differentiate between the general aging effects versus health behavior choices around physical activity. A trend of declining physical activity level with increasing age is well documented $[15,17,37]$. While this trend likely reflects a natural developmental change, with decline in functional reserve and physiologic capacity, the age at onset and rate of decline is highly variable. Thus, individuals of the same chronologic age can have very 
different functional or physiologic age, and thus be capable of being physically active. Further research is needed to separate the effects of aging from physical inactivity.

Our study also has many strengths that allowed us to address some of the gaps in QOL research in cancer survivors. By excluding cancer diagnoses within the first two years of the QOL assessment, we attempted to eliminate the effects of treatment on both QOL and lifestyle factors known to influence QOL [5-10]. Additionally, we excluded women who died within one year of the QOL assessment, thus minimizing the possibility of poor QOL due to impending death. We defined poor quality of life as more than one-half of a SD below the mean score of cancer-free women in our cohort, which equated to 4.1-5.9 points for each SF-36 scale. This amount equals or exceeds the estimate for minimally clinically important difference for this QOL instrument [28, 38]. Use of an internally defined threshold (versus population norms) allowed us to compare women of similar age and comorbidity status, which allowed us to separate the effects of age, comorbidity and cancer on QOL.

In conclusion, our findings suggest that among cancer survivors and cancer-free women, being physically inactive or becoming inactive is associated with worse mental and physical QOL, than being or staying physically active, independent of age, BMI, diet quality, and comorbidity status. These findings provide additional evidence on the importance of leisuretime physical activity in older women, especially for general health, vitality and physical function. Our findings support the need for interventions to help all older women maintain or regain a physically active lifestyle. Given the heterogeneity of the geriatric survivorship population in terms of health status, functional status, and physiologic reserve, multiple approaches are needed to promote physical activity in this population to achieve satisfactory levels of functioning and well-being.

\section{Supplementary Material}

Refer to Web version on PubMed Central for supplementary material.

\section{Acknowledgments}

We are grateful to the women who so generously gave of their time to participate in the Iowa Women's Health Study. Supported by the National Cancer Institute R01 CA039742.

\section{References}

1. de Moor JS, Mariotto AB, Parry C, Alfano CM, Padgett L, Kent EE, et al. Cancer Survivors in the United States: Prevalence across the Survivorship Trajectory and Implications for Care. Cancer Epidemiol Biomarkers Prev. 2013; 22(4):561-70. [PubMed: 23535024]

2. Smith AW, Reeve BB, Bellizzi KM, Harlan LC, Klabunde CN, Amsellem M, et al. Cancer, comorbidities, and health-related quality of life of older adults. Health Care Financ Rev. 2008; 29(4):41-56. [PubMed: 18773613]

3. Reeve BB, Potosky AL, Smith AW, Han PK, Hays RD, Davis WW, et al. Impact of cancer on health-related quality of life of older Americans. J Natl Cancer Inst. 2009; 101(12):860-8. [PubMed: 19509357]

4. Baker F, Haffer SC, Denniston M. Health-related quality of life of cancer and noncancer patients in Medicare managed care. Cancer. 2003; 97(3):674-81. [PubMed: 12548610] 
5. Mosher CE, Sloane R, Morey MC, Snyder DC, Cohen HJ, Miller PE, et al. Associations between lifestyle factors and quality of life among older long-term breast, prostate, and colorectal cancer survivors. Cancer. 2009; 115(17):4001-9. [PubMed: 19637244]

6. Schlesinger S, Walter J, Hampe J, von Schonfels W, Hinz S, Kuchler T, et al. Lifestyle factors and health-related quality of life in colorectal cancer survivors. Cancer Causes Control. 2014; 25(1):99110. [PubMed: 24158780]

7. Lynch BM, Cerin E, Owen N, Aitken JF. Associations of leisure-time physical activity with quality of life in a large, population-based sample of colorectal cancer survivors. Cancer Causes Control. 2007; 18(7):735-42. [PubMed: 17520334]

8. Thraen-Borowski KM, Trentham-Dietz A, Edwards DF, Koltyn KF, Colbert LH. Dose-response relationships between physical activity, social participation, and health-related quality of life in colorectal cancer survivors. J Cancer Surviv. 2013; 7(3):369-78. [PubMed: 23546822]

9. Blanchard CM, Courneya KS, Stein K. Cancer survivors' adherence to lifestyle behavior recommendations and associations with health-related quality of life: results from the American Cancer Society's SCS-II. J Clin Oncol. 2008; 26(13):2198-204. [PubMed: 18445845]

10. Trinh L, Plotnikoff RC, Rhodes RE, North S, Courneya KS. Associations between physical activity and quality of life in a population-based sample of kidney cancer survivors. Cancer Epidemiol Biomarkers Prev. 2011; 20(5):859-68. [PubMed: 21467240]

11. Fossa SD, Hess SL, Dahl AA, Hjermstad MJ, Veenstra M. Stability of health-related quality of life in the Norwegian general population and impact of chronic morbidity in individuals with and without a cancer diagnosis. Acta Oncol. 2007; 46(4):452-61. [PubMed: 17497312]

12. Vissers PA, Thong MS, Pouwer F, Zanders MM, Coebergh JW, van de Poll-Franse LV. The impact of comorbidity on Health-Related Quality of Life among cancer survivors: analyses of data from the PROFILES registry. J Cancer Surviv. 2013; 7(4):602-13. [PubMed: 23918453]

13. Inoue-Choi M, Lazovich D, Prizment AE, Robien K. Adherence to the World Cancer Research Fund/American Institute for Cancer Research recommendations for cancer prevention is associated with better health-related quality of life among elderly female cancer survivors. J Clin Oncol. 2013; 31(14):1758-66. [PubMed: 23569318]

14. Physical Activity Guidelines Advisory Committee. Physical Activity Guidelines Advisory Committee Report, 2008. Washington, DC: U.S. Department of Health and Human Services; 2008. p. G6-1-G6-31.

15. Bellizzi KM, Rowland JH, Jeffery DD, McNeel T. Health behaviors of cancer survivors: examining opportunities for cancer control intervention. J Clin Oncol. 2005; 23(34):8884-93. [PubMed: 16314649]

16. Coups EJ, Ostroff JS. A population-based estimate of the prevalence of behavioral risk factors among adult cancer survivors and noncancer controls. Prev Med. 2005; 40(6):702-11. [PubMed: 15850868]

17. Eakin EG, Youlden DR, Baade PD, Lawler SP, Reeves MM, Heyworth JS, et al. Health behaviors of cancer survivors: data from an Australian population-based survey. Cancer Causes Control. 2007; 18(8):881-94. [PubMed: 17638108]

18. Demark-Wahnefried W, Aziz NM, Rowland JH, Pinto BM. Riding the crest of the teachable moment: promoting long-term health after the diagnosis of cancer. J Clin Oncol. 2005; 23(24): 5814-30. [PubMed: 16043830]

19. Smith WA, Nolan VG, Robison LL, Hudson MM, Ness KK. Physical activity among cancer survivors and those with no history of cancer- a report from the National Health and Nutrition Examination Survey 2003-2006. Am J Transl Res. 2011; 3(4):342-50. [PubMed: 21904654]

20. Vallance JK, Courneya KS, Jones LW, Reiman T. Differences in quality of life between nonHodgkin's lymphoma survivors meeting and not meeting public health exercise guidelines. Psychooncology. 2005; 14(11):979-91. [PubMed: 15744764]

21. Mason C, Alfano CM, Smith AW, Wang CY, Neuhouser ML, Duggan C, et al. Long-term physical activity trends in breast cancer survivors. Cancer Epidemiol Biomarkers Prev. 2013; 22(6):115361. [PubMed: 23576689] 
22. Solberg Nes L, Liu H, Patten CA, Rausch SM, Sloan JA, Garces YI, et al. Physical activity level and quality of life in long term lung cancer survivors. Lung Cancer. 2012; 77(3):611-6. [PubMed: 22681871]

23. Alfano CM, Smith AW, Irwin ML, Bowen DJ, Sorensen B, Reeve BB, et al. Physical activity, longterm symptoms, and physical health-related quality of life among breast cancer survivors: a prospective analysis. J Cancer Surviv. 2007; 1(2):116-28. [PubMed: 18648952]

24. Morey MC, Snyder DC, Sloane R, Cohen HJ, Peterson B, Hartman TJ, et al. Effects of home-based diet and exercise on functional outcomes among older, overweight long-term cancer survivors: RENEW: a randomized controlled trial. JAMA. 2009; 301(18):1883-91. [PubMed: 19436015]

25. Vallance JK, Courneya KS, Plotnikoff RC, Yasui Y, Mackey JR. Randomized controlled trial of the effects of print materials and step pedometers on physical activity and quality of life in breast cancer survivors. J Clin Oncol. 2007; 25(17):2352-9. [PubMed: 17557948]

26. Folsom AR, Kaye SA, Prineas RJ, Potter JD, Gapstur SM, Wallace RB. Increased incidence of carcinoma of the breast associated with abdominal adiposity in postmenopausal women. Am J Epidemiol. 1990; 131(5):794-803. [PubMed: 2138863]

27. Walters SJ, Munro JF, Brazier JE. Using the SF-36 with older adults: a cross-sectional communitybased survey. Age Ageing. 2001; 30(4):337-43. [PubMed: 11509313]

28. Norman GR, Sloan JA, Wyrwich KW. Interpretation of changes in health-related quality of life: the remarkable universality of half a standard deviation. Med Care. 2003; 41(5):582-92. [PubMed: 12719681]

29. Willett WC, Sampson L, Browne ML, Stampfer MJ, Rosner B, Hennekens CH, et al. The use of a self-administered questionnaire to assess diet four years in the past. Am J Epidemiol. 1988; 127(1):188-99. [PubMed: 3337073]

30. WCRF/AICR. Food, Nutrition, Physical Activity, and the Prevention of Cancer: a Global Perspective. Washington, DC: World Cancer Research Fund/American Institute for Cancer Research; 2007.

31. Inoue-Choi M, Robien K, Lazovich D. Adherence to the WCRF/AICR guidelines for cancer prevention is associated with lower mortality among older female cancer survivors. Cancer Epidemiol Biomarkers Prev. 2013; 22(5):792-802. [PubMed: 23462914]

32. Lazovich D, Robien K, Cutler G, Virnig B, Sweeney C. Quality of life in a prospective cohort of elderly women with and without cancer. Cancer. 2009; 115(18 Suppl):4283-97. [PubMed: 19731348]

33. Hewitt M, Rowland JH, Yancik R. Cancer survivors in the United States: age, health, and disability. J Gerontol A Biol Sci Med Sci. 2003; 58(1):82-91. [PubMed: 12560417]

34. Blair CK, Morey MC, Desmond RA, Cohen HJ, Sloane R, Snyder DC, et al. Light-Intensity Activity Attenuates Functional Decline in Older Cancer Survivors. Med Sci Sports Exerc. 2014

35. Voskuil DW, van Nes JG, Junggeburt JM, van de Velde CJ, van Leeuwen FE, de Haes JC. Maintenance of physical activity and body weight in relation to subsequent quality of life in postmenopausal breast cancer patients. Ann Oncol. 2010; 21(10):2094-101. [PubMed: 20357033]

36. Blanchard CM, Baker F, Denniston MM, Courneya KS, Hann DM, Gesme DH, et al. Is absolute amount or change in exercise more associated with quality of life in adult cancer survivors? Prev Med. 2003; 37(5):389-95. [PubMed: 14572423]

37. Tucker JM, Welk GJ, Beyler NK. Physical activity in U.S.: adults compliance with the Physical Activity Guidelines for Americans. Am J Prev Med. 2011; 40(4):454-61. [PubMed: 21406280]

38. Hays RD, Morales LS. The RAND-36 measure of health-related quality of life. Ann Med. 2001; 33(5):350-7. [PubMed: 11491194] 


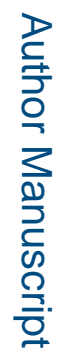

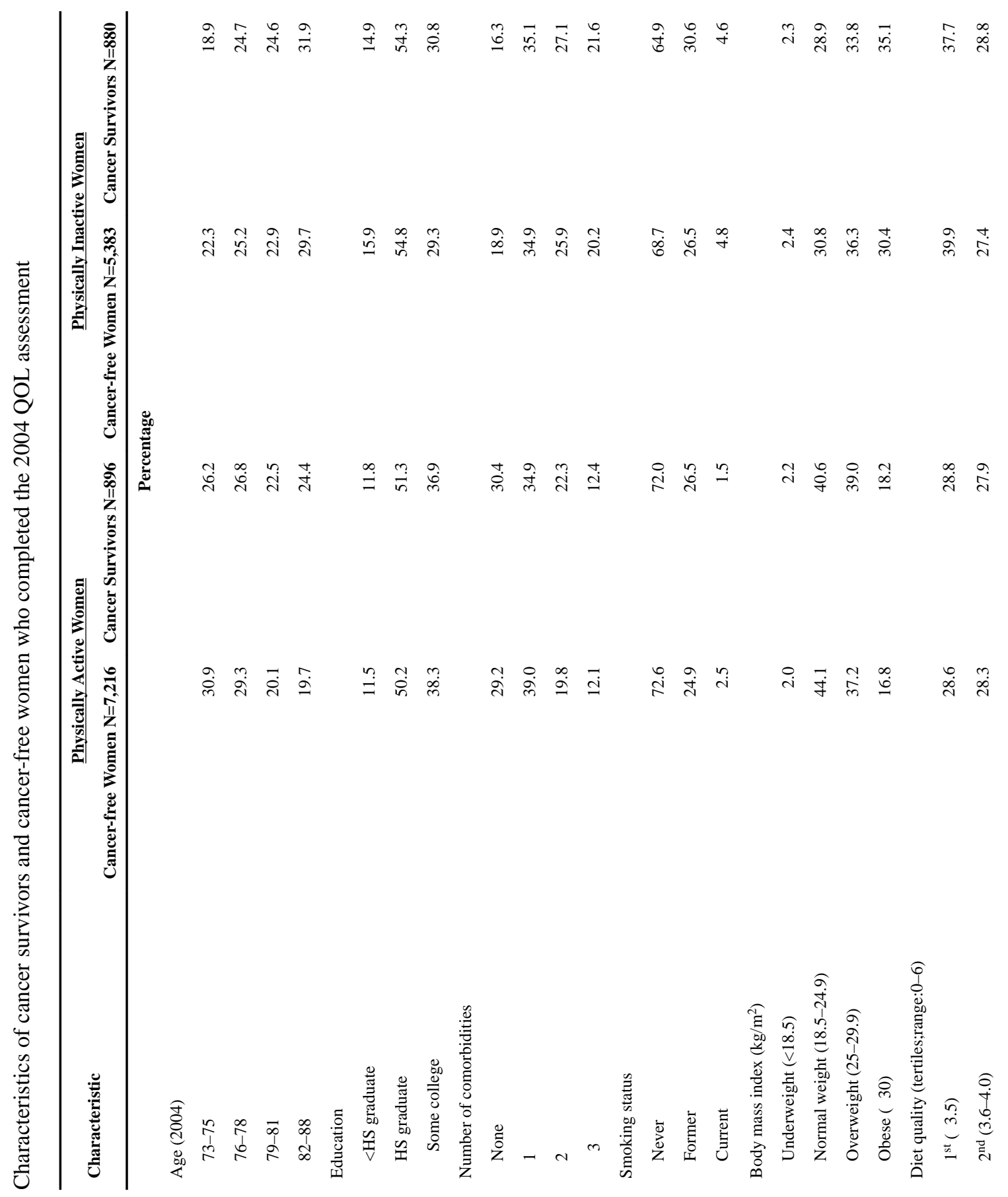

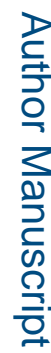

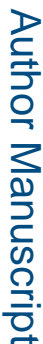

J Cancer Surviv. Author manuscript; available in PMC 2017 February 01. 
Blair et al.

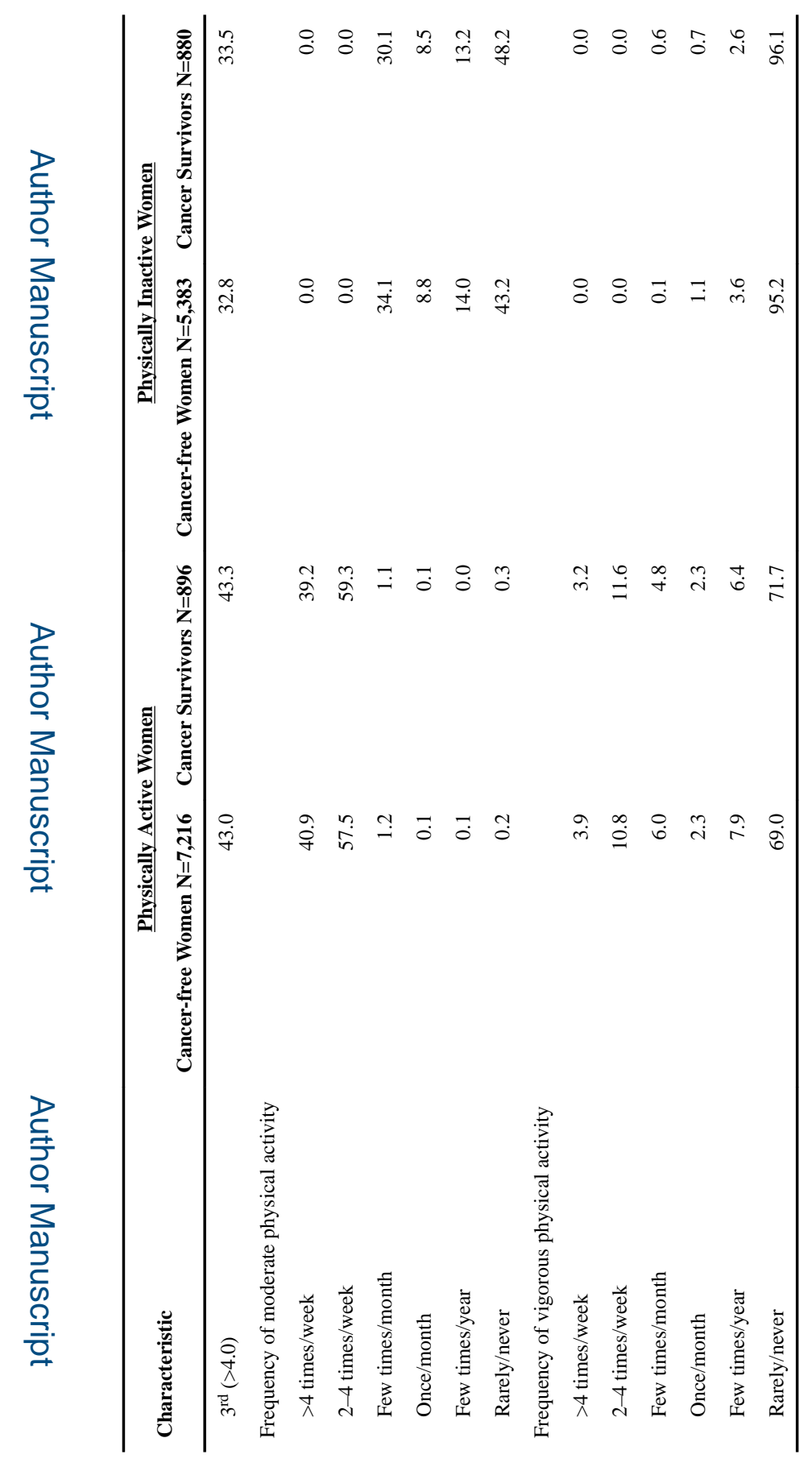

로을 


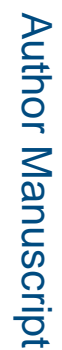

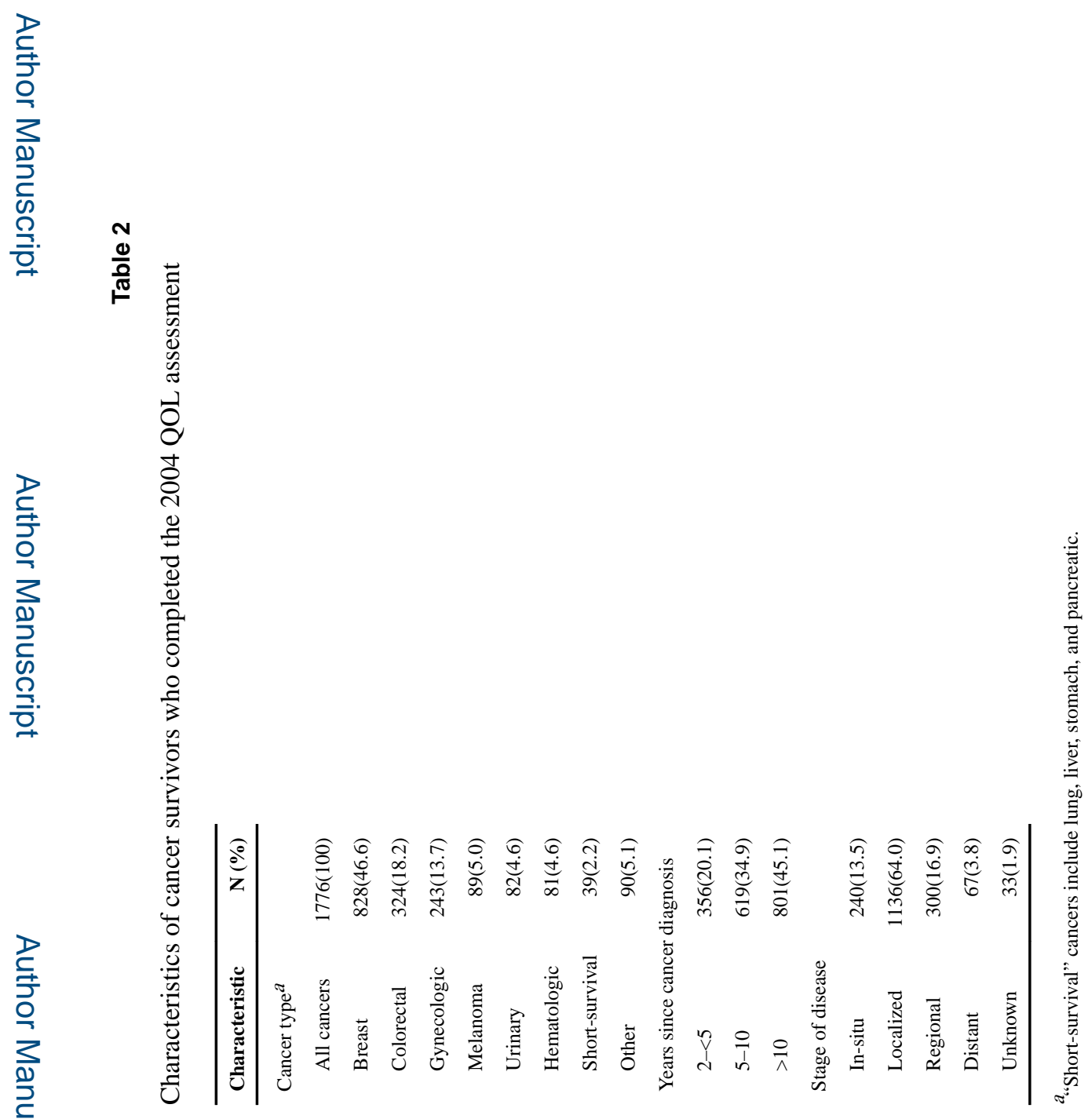

J Cancer Surviv. Author manuscript; available in PMC 2017 February 01. 


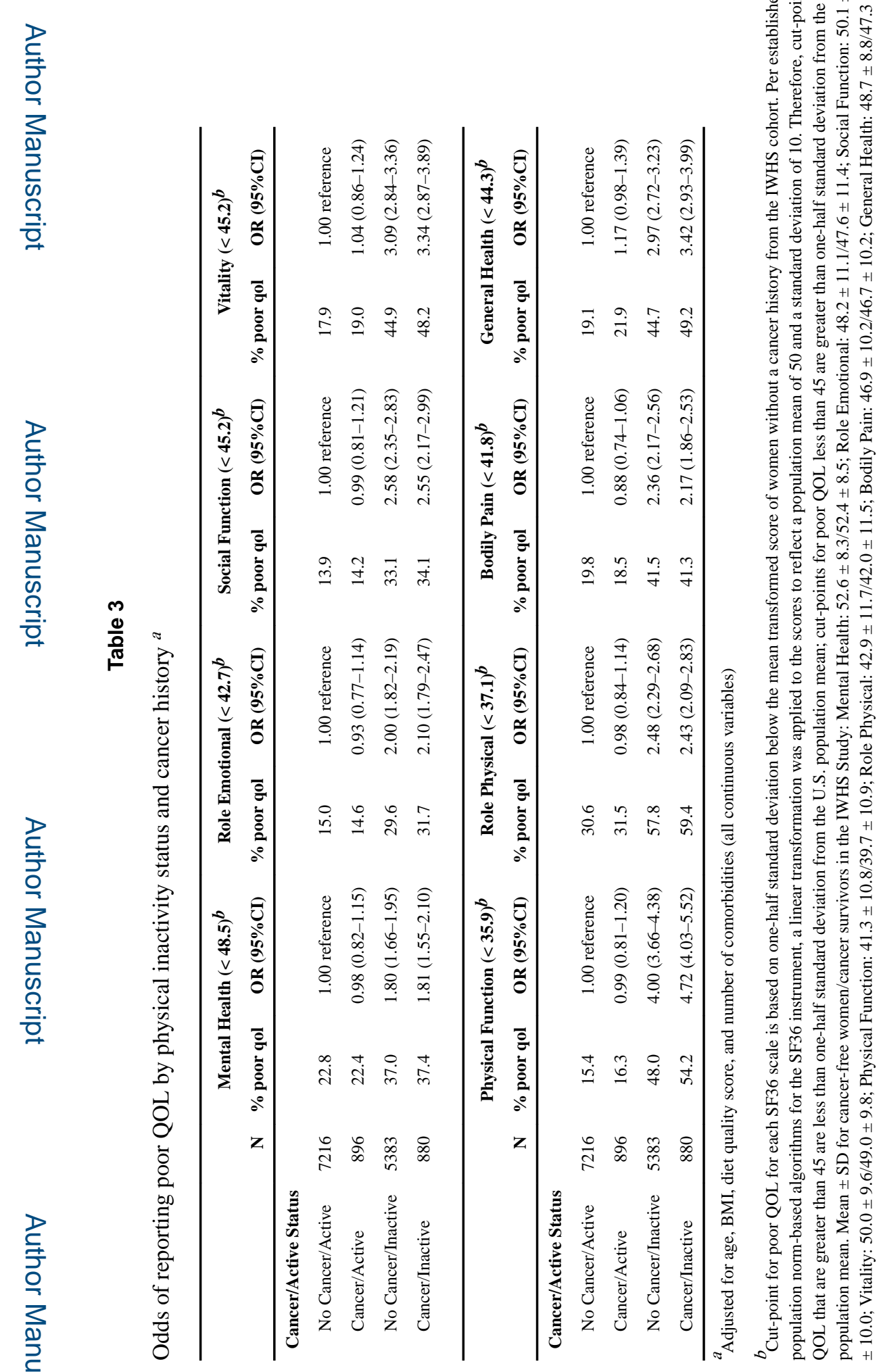

J Cancer Surviv. Author manuscript; available in PMC 2017 February 01. 


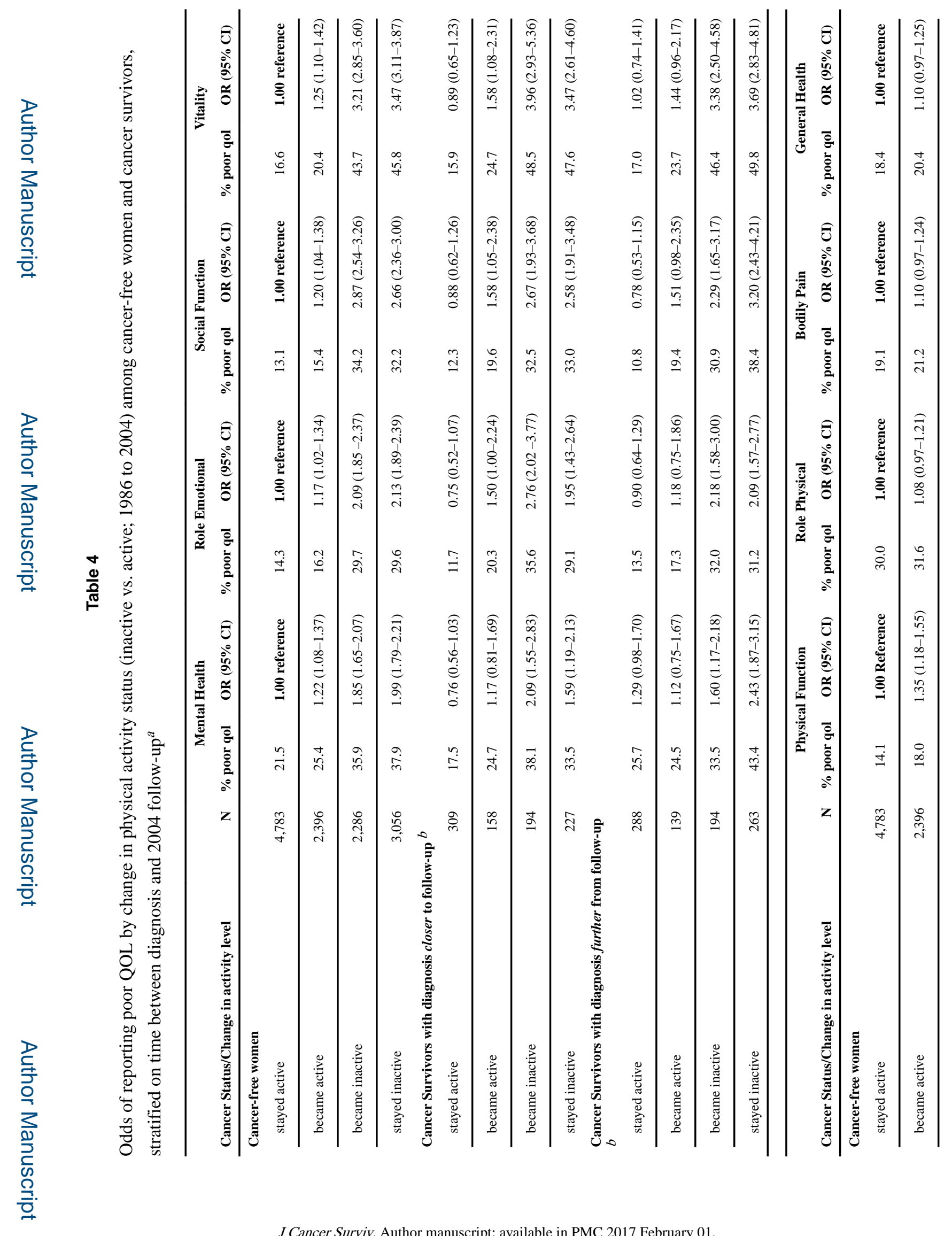


Blair et al.

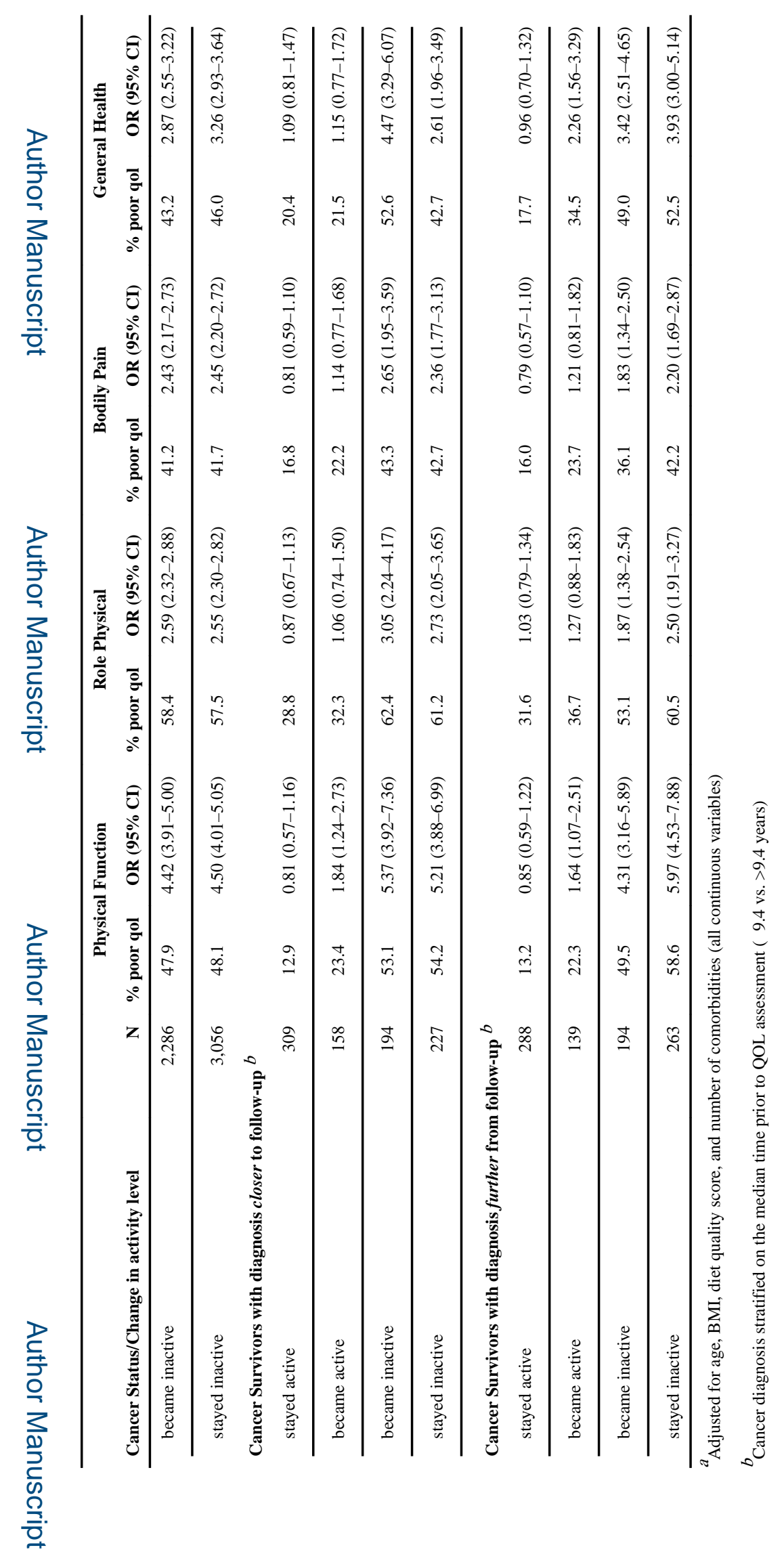

J Cancer Surviv. Author manuscript; available in PMC 2017 February 01. 